\title{
Resistance of Candida glabrata to drugs and the host immune system
}

\author{
Khirun Musa ${ }^{1}$, Mowaffaq A Ahmed ${ }^{1}$, Siti NM Shahpudin ${ }^{1}$, Laina ZM Kamal ${ }^{1}$, Rosline Sandai ${ }^{2}$, Yasser Tabana ${ }^{3}$ and Doblin Sandai ${ }^{1 *}$ \\ ${ }^{1}$ Infectomics Cluster, Advanced Medical and Dental Institute, Universiti Sains Malaysia, Bertam Penang, Malaysia \\ ${ }^{2}$ Faculty of Languages and Communication, Sultan Idris University of Education, Perak, 35900 Malaysia \\ ${ }^{3}$ Faculty of Pharmacy and Pharmaceutical Sciences, University of Alberta, Edmonton, Alberta, Canada
}

\begin{abstract}
Background: Candida glabrata is a ubiquitous organism and this species is one of the normal floras that commonly found in oral cavity, gastrointestinal and vaginal tract. However, they can be pathogenic and caused serious infection that more likely to occur in immunocompromised patients. Widespread and increased usage of immunosuppressive therapies with the additional of broad-spectrum antibiotic treatment can also lead to the significant increment of Candida infections. Candida species can cause nosocomial infection that associated with alarming high mortality rate worldwide. C. glabrata is one of the Candida species that raise an attention of a researcher due to their high resistance to certain drugs and antifungal as well as host immune system.
\end{abstract}

Objective: To investigate certain aspects of the C. glabrata that contributes to their resistance to drugs and as host in immune system.

Results: Microorganisms including Candida have an ability to evolve in order to sustain their survival and it is creating a new microbial community that are more resistant. C. glabrata possessed several strategies for their resistance including production of biofilm, genomic plasticity as well as chromatin alteration. On the other hand, their resistance may cause by the mutation in certain gene. Besides, they also use a variety of mechanisms that enable their adaptation works successfully and their survival as host in immune system.

Conclusion: A significant number of researches have been conducted to elucidate resistant mechanism of $C$. glabrata thus, much information has been gained on how their resistant mechanism works and has enhanced our knowledge on the virulence and resistance mechanisms associated with $C a n d i d a$ species especially $C$. glabrata. Further studies and investigation in this area are crucial particularly on the identification and development of new targets for novel therapeutics against these recently emerged pathogens.

\section{Introduction}

Candida species are known as one of the normal flora in oral cavity, gastrointestinal tract, and vaginal tracts [1-7] of healthy individuals but rarely causing serious infection in humans [5,8-11]. However, widespread and increased usage of immunosuppressive therapies together with the additional broad-spectrum antibiotic treatment, have led to a significant increment infections caused by C. glabrata that associated with a high mortality rate $[5,8,9,11,12]$.

Candidiasis represent one of the main causes for the nosocomial infection worldwide [1,3,7,9-11,13-16]. Candida infections are more likely to occur in immunocompromised patients such as patients in intensive care, post-surgical patients, HIV-positive people, patient under chemotherapy treatment and diabetic patient $[1,3,7,8,11,13$, 14,16-18,19]. Candida genus comprises over 150 heterogeneous species, but only a minority are associated to human candidosis, as $65 \%$ of Candida species are unable to grow at a temperature of $37^{\circ} \mathrm{C}$ thus prevent them from being pathogenic to the human [3-5] and more than 17 different Candida species reported to be etiological agents of human infections $[4,17,20,21]$. The most common species that cause infection in human is Candida albicans $[2,3,5,10,11,17,21,22]$ and most common non-albicans Candida species that play a role as a causative infectious agent in human is C. glabrata [1,4,5,7-11,17,20,22-25].

\section{Pathogenicity of Candida}

The pathogenicity of Candida species depends on virulence factors such as adherence to epithelial and endothelial cells [3,5,11,22], ability to evade host defences [2], biofilm formation on host tissue or on medical devices [2,5] production of tissue-damaging hydrolytic enzymes such as proteases, phospholipases, and hemolysins [2-5,11,22] hypha and pseudohypha formation [3-5,11,22] and phenotypic switching [11].

Adherence is an extremely important virulence factor, even though the actual adherence property may be influenced by other virulence $[5,11]$. Initiation of Candida infection is its adherence of the organism to host and/or medical-device surfaces, since it is required for their initial colonization and for Candida species they also can adhere to the surfaces of medical devices and form biofilm [5,9,26]. Adhesion is an extremely important step in the infection process, and several factors implicated in influencing adhesion are microbial host, abiotic surface properties (such as cell-surface hydrophobicity and cell-

*Correspondence to: Doblin Sandai, Infectomics Cluster, Advanced Medical and Dental Institute, Universiti Sains Malaysia, Bertam Penang, Malaysia, E-mail: doblin@usm.my

Received: November 26, 2018; Accepted: December 11, 2018; Published: December 15, 2018 
wall composition) and profile of cell wall proteins [5,6, 9,18,22,26]. Microbial biofilms were produced when organisms start to adhere to a surface and produce extracellular polymers that provide a structural matrix and facilitate further adhesion [7].

On the other hand, biofilm formation is another crucial virulence factors for a number of Candida species as their defence mechanism against nutritional and environmental stress [16]. Biofilm formation limiting the penetration of substances through the matrix and protecting cells from host immune responses as well as gives a significant resistance to antifungal therapy $[4,5,13]$. Biofilms can grow on any biotic or abiotic moist surface and considered as a representative of the microorganisms growth form as well as forming a protection for their development $[2,5,13]$. The ability of Candida species to form drugresistant biofilms is an important factor that lead to their contribution in human disease $[2,4]$. Biofilm formation involve several different phases including initial adherence, colonization, proliferation, maturation and ultimately dispersion [13]. C. albicans biofilm formation can be divided into three developmental phases that are adherence of yeast cells to the device surface (early phase), formation of a matrix with from yeast to hyphal forms (intermediate phase), and increase in the matrix material taking on a three-dimensional architecture (maturation phase) $[7,16]$.

Moreover, degradative enzymes also play an important role in virulence [2] and activity of these enzymes has been reported to be associated with Candida adhesion, cell damage and invasion of host tissue [9]. Destruction of host tissues by Candida species may be facilitated by the release of hydrolytic enzymes [2,5] since they have ability to produce and secrete several hydrolytic enzymes, including proteases, phospholipases (PLs) and haemolysins [2,5,11,22]. Phospholipases (PLs) production can lead to host cell membrane damage, promoting cell damage or expose receptors to facilitate adherence by hydrolyzing phospholipids into fatty acids [4,5]. Several studies of Non-Candida albicans Candida (NCAC) species reported that they have the ability to produce extracellular phospholipids at lower levels as compared to C. albicans and because it is a strong strain-dependent $[5,9]$.

\section{Candida glabrata}

Among the Candida species, C. glabrata is one species that raises a special concern because of its inherent resistance to certain antifungal agents and frequent isolation as a non-albicans Candida species (25,7-11,20,26,27). C. glabrata grows and forms shining, smooth, and cream coloured colonies, which are indistinguishable from other Candida species, except for their size which is smaller. C. glabrata cells are approximately $1-4 \mu \mathrm{m}$ in size which are remarkably smaller than C. albicans that have size around 4-6 $\mu \mathrm{m}$ [4]. They can cause different clinical manifestations, from minor infections to life-threatening invasive infections [3].

The most highlighted distinguishing characteristic of C. glabrata that make it unique as compared to C. albicans and another NACS is its haploid genome $[1,3-5,8,11,23]$. Another different characteristic of C. glabrata is that they are not polymorphic and are growing only as blastospores $[4,5,8,9,11]$. The cell surface of $C$. glabrata can exhibits a degree of hydrophobicity that facilitate in their adhesion $[2,5,6,8,9,18,22,23]$ and additionally, compared with C. albicans, C. glabrata has been reported to have a higher aptitude to adhere to denture acrylic surfaces $[9,22]$ C. glabrata also have an ability to produce hemolysins that can degrade haemoglobin in order to get an iron from host cells for metabolic processes [4,9].

\section{Resistance mechanism of Candida glabrata}

The ability of Candida species to form a drug-resistant biofilms is the most important factor that associated with a high level of antimicrobial resistance that relates to many human diseases and high mortal rate $[1,2,4,5,7,8,24,26,27]$. Fungal cells have evolved different mechanisms, including target alteration, reducing uptake and active extrusion for their sustainability by allowing protection of cell integrity, and are coordinated with stress response signalling pathways [1].

Different from another Candida species, C. glabrata do not produce proteases but they produces phospholipases that promotes a greater and powerful interface to give an effective invasion of the tissues involved $[4,8]$. Attachment of Candida cells to the host commonly initiated by certain factors such as hydrophobicity and electrostatic forces that is promoted by adhesins which recognize ligands such as proteins, fibrinogen and fibronectin [2,5,19]. In C. glabrata, adhesins is encoded by the EPA (epithelial adhesin) gene family $[1,4,5,8,9,23,28]$. Previous study has shown that the deletion of EPA1 gene can be reduced in vitro adhesion $[4,5,8,9,23]$. Other than that, EPA6 that expressed in vivo during urinary infection but not in vitro showed that C. glabrata is able to adapt to different environment for their sustainability $[4,5,8,9,23]$.

Besides, biofilm forming ability in C. glabrata give an advantages by allowing them to evade host immune mechanisms, resist antifungal treatment, and withstand the competitive pressure from other microorganisms $[2,4,5]$. C. glabrata displays the lowest biofilm metabolic activity, despite having the highest number of biofilm cultivable cells as well as having less total biomass and its strains show similar biofilm forming ability, in opposition to C. parapsilosis and $C$. tropicalis $[2,4,5,9]$. Other than that, their biofilm production has been reported to have relatively higher quantities of protein and carbohydrate in contrast with other NCACs [2,4,5,9]. Different mechanisms may be involved in the resistance of C. glabrata through production of biofilms such as high density of cells within the biofilm, effects of the biofilm matrix, decreased growth rate and nutrient limitation, expression of resistance genes, particularly those encoding efflux pumps and presence of "persister" cells [4].

Similar to other Candida species, C. glabrata have ability to adapt to their environmental alterations in the presence of antifungal agents as well as to provide protection against antimicrobial therapies $(1,2,4,5,7,8,24,26,27)$. Azoles, like fluconazole, itraconazole, ketoconazole, miconazole, voriconazole, posaconazole, and rosaconazole, are antifungal that have an ability to block the ergosterol biosynthesis by the inhibition of lanosterol $14 \alpha$-demethylase, leading to a decrement in ergosterol production $(1,3-5,8,19)$.

C. glabrata also have an ability to develop azole resistance at a relatively high frequency and major mechanism involved is overexpression of ATP binding cassette (ABC) transporters such as CDR1 (CgCDR1), CgCDR2, and CgSNQ2 [28]. Moreover, this type of antifungal is responsible for the lower ability to build and renew sterols in the cellular membranes, changing the membrane fluidity and function of important processes such as signalling, transport, exocytosis, and endocytosis [4]. Other than azole, polyenes (amphotericin B, nystatin, and pimaricin) that link to the ergosterol molecules are enable in inducing pores on the cell wall and increasing cell permeability [2 4,8]. On the other hand, antimetabolite antifungal drugs, such as 5- fluorocytosine have an abilityto use distinct means to inhibit the growth of fungal pathogens by inhibiting RNA function [4].

Apart from that, genomic plasticity plays a critical role for adaptation of the C. glabrata to environmental changes, such as host 
responses and anti- fungal drug pressure [4]. Genomic plasticity in C. glabrata, has caused several genes such as gene that encode for galactose and sucrose assimilation, phosphate, nitrogen, and sulphur metabolism, as well as thiamine, pyridoxine, and nicotinic acid biosynthesis lost from the genome [4]. Previous study has shown that chromosomal aberrations and functional adaptations may occur during infection, under antimicrobial therapy and also under laboratory conditions hindered by extreme selective pressures $[4,8]$. Chromatin alterations could happen in C. glabrata as essential strategies for their survival that facilitates a reprogramming of cellular energy metabolism in macrophage-internalized cells, and provide protection against DNA damage $[4,23]$.

\section{Candida glabrata and host immune response}

In human body, innate immunity act as a first defence in combating antigen and pathogen [29,30]. Innate immune system can provoke pathogen-specific responses by recognizing microbes based on their pathogen-associated molecular patterns (PAMPs) [29-31]. It is common for macrophages to be involved in an early stage of infection by detecting and phagocytizing infectious pathogen since they express a different receptors that are able to detect oneself particles [29]. C. glabrata invasion is one of the pathogen that triggers both macrophages and cytokine secretion [29,30,32-34]. Previous study has also shown that polymorphonuclear leukocytes and/or macrophage antifungal activities is modulated by cytokines [34]. Phagosome is formed when the phagocyte (such as leukocytes or neutrophils, dendritic cells and macrophages) wraps a portion of its plasma membrane around the pathogenic particle. It is followed by plasma membrane fusion at the tip of the particle and finally newly formed membrane bag containing the particle were ingested [33]. Phagosome activity of macrophages poses a nutritional challenge for the pathogens where alternative sources of carbon, nitrogen, and trace elements must be used to promote growth [29].

C. glabrata has been described as one of the pathogen that has an ability to survive from phagocytosis by macrophages and replicates within macrophages $[29,30,32]$. Upon to response to macrophages, C. glabrata up-regulates methylcitrate cycle genes thus enables the degradation of fatty acid chains and allowing lipids to be used as an alternative carbon source as well as up-regulates lysine biosynthesis [29]. Previous study has reported that from screening a set of 433 deletion mutant, 23 genes that responsible to resist them from being killed by macrophages has been identified [32]. There are several factors for $C$. glabrata-phagocyte interactions including uptake by macrophages, replication within macrophages, phagosomal $\mathrm{pH}$, and recognition of mutant cells by macrophages as indicated by production of reactive oxygen species and tumour necrosis factor alpha (TNF- $\alpha$ ) [32]. Following the phagocytosis, the production of reactive oxygen species (ROS) by macrophages can kill microorganisms or macrophage antimicrobial response but C. glabrata is known to have an ability to inhibit ROS [30,32]. Production of ROS is modulated by the macrophage NADPH oxidase complex which is assembled at the cytoplasmic and phagosomal membrane and generates superoxide, hydroxyl anions and hydroxyl radicals [30]. C. albicans and C. glabrata are able to actively limit phagosome maturation in macrophages to prevent acidification and limiting hydrolytic attack and for C. glabrata they are not always virulent. It shows that other mechanisms may exist to allow their survival, replication, and escape from within macrophages [29].

In general, fungal pathogens have an ability to control their metabolism in the glucose-deficient environment inside the macrophage and they also enable to adjust their expression profile of the genes involved in the employment of alternative carbon sources for their survival [4]. Genes of the glycolytic pathway are downregulated, whereas genes that encode for proteins that are needed for gluconeogenesis, $\beta$-oxidation, glyoxylate cycle, autophagy, peroxisome proliferation, and amino and acetate transports are up-regulated [4]. Furthermore, secretion of enzymes, such as proteases, phospholipases, lipases, and hemolysins, by the yeasts facilitate them to survive and replicate within macrophages and it also can destroy the epithelium [4]. Even though C. glabrata have shown that they do not produce proteases, there is one study shows that this species is enabling to produce proteinase. However, the type of proteinase produced are not specified [4].

A variety of mechanisms are evolved by pathogen in order to allow their successful adaptation and survival in the host including the ability to sustain long-term carbon and iron starvation upon phagocytosis by macrophages, as well as a low-pH environment in the vagina or phagolysosomes, and increase resistance to nitrosative and oxidative stresses [1]. Similar to all Candida species, C. glabrata possess the ability to adapt to the environmental alterations in the presence of antifungal agents in order to provide protection against antimicrobial therapies [4].

\section{Conclusion}

A significant number of researches have been conducted to elucidate resistant mechanism of C. glabrata thus, much information has been gained on how their resistant mechanism works and has enhanced our knowledge on the virulence and resistance mechanisms associated with Candida species especially C. glabrata. Further studies and investigation in this area are crucial particularly on the identification and development of new targets for novel therapeutics against these recently emerged pathogens.

\section{Acknowledgement}

KM was funded and would like to thank Universiti Sains Malaysia for providing financial support from the University Individual (RUI) grant no: 1001/CIPPT/812196.

\section{References}

1. Kołaczkowska A, Kołaczkowski M (2016) Drug Resistance Mechanisms and Their Regulation in non-albicans Candida Species. J Antimicrob Chemother 71: 1438-1450. [Crossref]

2. Sardi JC, Scorzoni L, Bernardi T, Fusco-Almeida AM, Mendes Giannini MJ (2013) Candida Species: Current Epidemiology, Pathogenicity, Biofilm Formation, Natura Antifungal Products and New Therapeutic Options. J Med Microbiol 62: 10-24. [Crossref]

3. Szweda P, Gucwa K, Romanowska E, Dzierzanowska-Fangrat K, Naumiuk $\iota$, et al (2015) Mechanisms of Azole Resistance among Clinical Isolates of Candida glabrata in Poland. J Med Microbiol 64: 610-619. [Crossref]

4. Rodrigues CF, Silva S, Henriques M (2013) Candida glabrata: A Review of Its Features and Resistance. Eur J Clin Microbiol Infect Dis 33: 673-688. [Crossref]

5. Silva S1, Negri M, Henriques M, Oliveira R, Williams DW, et al. (2012) Candida glabrata, Candida parapsilosis and Candida tropicalis: Biology, Epidemiology, Pathogenicity and Antifungal Resistance. FEMS Microbiol Rev 36: 288-305. [Crossref]

6. Chaffin WL (2008) Candida albicans Cell Wall Proteins. Microbiol Mol Biol Rev 72 495-544. [Crossref]

7. Kojic EM, Darouiche RO (2004) Candida Infections of Medical Devices. Clin Microbiol Rev 17: 255-267. [Crossref]

8. Rodrigues CF, Rodrigues ME, Silva S, Henriques M (2017) Candida glabrata Biofilms: How Far Have We Come? J Fungi (Basel) 3: 11. [Crossref]

9. Silva S, Negri M, Henriques M, Oliveira R, Williams DW, et al. (2011) Adherence and Biofilm Formation of Non-Candida albicans Candida Species. Trends Microbiol 19: 241-247. [Crossref] 
10. Malani A, Hmoud J, Chiu L, Carver PL, Bielaczyc A, et al. (2005) Candida glabrata Fungemia: Experience in a Tertiary Care Center. Clin Infect Dis 41: 975-981. [Crossref]

11. Fidel PL, Vazquez JA, Sobel JD (1999) Candida glabrata: Review of Epidemiology, Pathogenesis, and Clinical Disease with Comparison to C. albicans. Clin Microbiol Rev 12: 80-96. [Crossref]

12. Hajjeh RA, Sofair AN, Harrison LH, Lyon GM, Arthington-Skaggs BA, et al. (2004) Incidence of Bloodstream Infections Due to Candida Species and In Vitro Susceptibilities of Isolates Collected from 1998 to 2000 in a Population-Based Active Surveillance Program. J Clin Microbiol 42: 1519-1527. [Crossref]

13. Uppuluri P, Pierce CG, López-Ribot JL (2009) Candida albicans Biofilm Formation and Its Clinical Consequences. Future Microbiol 4: 1235-1237. [Crossref]

14. Vazquez JA, Dembry LM, Sanchez V, Vazquez MA, Sobel JD, et al. (1998) Nosocomial Candida glabrata Colonization: An Epidemiologic Study. J Clin Microbiol 36: 421426. [Crossref]

15. Reagan DR, Pfaller MA, Hollis RJ, Wenzel RP (1990) Characterization of the Sequence of Colonization and Nosocomial Candidemia Using DNA Fingerprinting and a DNA Probe. J Clin Microbiol 28: 2733-2738. [Crossref]

16. Sandai D, Tabana YM, Ouweini AE, Ayodeji IO (2016) Resistance of Candida albicans Biofilms to Drugs and the Host Immune System. Jundishapur J Microbiol 9: 1-7. [Crossref]

17. Pfaller MA, and Diekema DJ (2007) Epidemiology of Invasive Candidiasis: A Persistent Public Health Problem. Clin Microbiol Rev 20:133-163. [Crossref]

18. Mariana H, Gasparetto K, Azeredo J (2002) Experimental Methodology to Quantify Candida albicans Cell Surface Hydrophobicity. Biotechnology Letters 24: 1111-1115. [Crossref]

19. Samaranayake LP, Fidel PL, Naglik JR, Sweet SP, Teanpaisan R, et al. (2002) Fungal Infections Associated with HIV Infection. Oral Dis 8:151-160. [Crossref]

20. Pfaller MA, Diekema DJ (2004) Rare and Emerging Opportunistic Fungal Pathogens: Concern for Resistance beyond Candida albicans and Aspergillus fumigatus. $J$ Clin Microbiol 42: 4419-4431. [Crossref]

21. Hazen KC (1995) New and Emerging Yeast Pathogens. Clin Microbiol Rev 8: 462-478. [Crossref]

22. Luo G, Samaranayake LP (2002) Candida glabrata, an Emerging Fungal Pathogen, Exhibits Superior Relative Cell Surface Hydrophobicity and Adhesion to Denture Acrylic Surfaces Compared with Candida albicans. APMIS 110: 601-610. [Crossref]
23. d'Enfert C, Janbon G (2015) Biofilm Formation in Candida glabrata: What Have We Learnt from Functional Genomics Approaches? FEMS Yeast Res 16: 1-13. [Crossref]

24. Jain N, Kohli R, Cook E, Gialanella P, Chang T, et al. (2007) Biofilm Formation by and Antifungal Susceptibility of Candida Isolates from Urine. Appl Environ Microbiol 73: 1697-1703. [Crossref]

25. Bennett JE, Izumikawa K, Marr KA (2004) Mechanism of Increased Fluconazole Resistance in Candida glabrata during Prophylaxis Mechanism of Increased Fluconazole Resistance in Candida glabrata during Prophylaxis. Antimicrob Agents Chemother 48: 1773-1777. [Crossref]

26. Finkel JS, Mitchell AP (2001) Genetic Control of Candida albicans Biofilm Development. Nat Rev Microbiol 9: 109-118. [Crossref]

27. Baillie GS, Douglas LJ (2000) Matrix Polymers of Candida Biofilms and Their Possible Role in Biofilm Resistance to Antifungal Agents. J Antimicrob Chemother 46: 397-403. [Crossref]

28. Ferrari S, Sanguinetti M, De Bernardis F, Torelli R, Posteraro B, et al. (2011) Loss of Mitochondrial Functions Associated with Azole Resistance in Candida glabrata Results in Enhanced Virulence in Mice. Antimicrob Agents Chemother 55: 18521860. [Crossref]

29. Gilbert AS, Wheeler RT, May RC (2015) Fungal Pathogens: Survival and Replication within Macrophages. Cold Spring Harb Perspect Med 5: 1-14. [Crossref]

30. Kasper L, Seider K, Hube B (2015) Intracellular Survival of Candida glabrata in Macrophages: Immune Evasion and Persistence. FEMS Yeast Res 15: 1-12. [Crossref]

31. Wheeler RT, Fink GR (2006) A Drug-Sensitive Genetic Network Masks Fungi from the Immune System. PLoS Pathog 2: 328-339. [Crossref]

32. Seider K, Gerwien F, Kasper L, Allert S, Brunke S, et al. (2014) Immune Evasion, Stress Resistance, and Efficient Nutrient Acquisition Are Crucial for Intracellular Survival of Candida glabrata within Macrophages. Eukaryot Cell 13: 170-183. [Crossref]

33. Haas A (2007) The Phagosome: Compartment with a License to Kill. Traffic 8: 311330. [Crossref]

34. Brieland J, Essig D, Jackson C, Frank D, Loebenberg D, et al. (2001) Comparison of Pathogenesis and Host Immune Responses to Candida glabrata and Candida albicans in Systemically Infected Immunocompetent Mice Comparison of Pathogenesis and Host Immune Responses to Candida glabrata and Candida albicans in Systemically Infected Immunocompetent Mice. Infect Immun 69: 5046-5055. [Crossref]

Copyright: (C2018 Musa K. This is an open-access article distributed under the terms of the Creative Commons Attribution License, which permits unrestricted use, distribution, and reproduction in any medium, provided the original author and source are credited. 